\title{
Is $\mathcal{P}(\omega)$ a subalgebra?
}

by

\begin{abstract}
Alan Dow (Charlotte, NC) and Ilijas Farah (North York and Beograd)
Dedicated to the memory of Murray Bell
\end{abstract}

\begin{abstract}
We consider the question of whether $\mathcal{P}(\omega)$ is a subalgebra whenever it is a quotient of a Boolean algebra by a countably generated ideal. This question was raised privately by Murray Bell. We obtain two partial answers under the open coloring axiom. Topologically our first result is that if a zero-dimensional compact space has a zero-set mapping onto $\beta \mathbb{N}$, then it has a regular closed zero-set mapping onto $\beta \mathbb{N}$. The second result is that if the compact space has density at most $\omega_{1}$, then it will map onto $\beta \mathbb{N}$ if it contains a zero-set that maps onto $\beta \mathbb{N}$.
\end{abstract}

1. Introduction. As mentioned in the abstract, Murray Bell raised the following question: if a compact zero-dimensional space has a zero-set which maps onto $\beta \mathbb{N}$, must the space map onto $\beta \mathbb{N}$ ? It was proved in [Dow97] that a negative answer follows from the Continuum Hypothesis (see also [vM01] for a simpler proof of a stronger result). In this paper, we obtain partial positive answers under the Open Coloring Axiom.

The question can be cast in Boolean algebraic language as follows. If $I$ is a countably generated ideal of a Boolean algebra $B$, and if $B / I$ contains $\mathcal{P}(\omega)$, must $B$ itself contain $\mathcal{P}(\omega)$ ? The following definition will be useful in formulating our partial result and was introduced in [Dow97].

Definition 1.1. Let $B$ be a Boolean algebra. We say that a family $A \subset B$ is separated from a family $C$ if there is a $b \in B$ such that $a \leq b$ for all $a \in A$ and $b \wedge c=0$ for all $c \in C$. We say that a family $A$ is completely separated if for each $C \subset A, A \backslash C$ is separated from $C$.

We will prove that if OCA holds, then a Boolean algebra $B$ will have an infinite completely separated family if it has a countably generated ideal $I$

2000 Mathematics Subject Classification: Primary 54A35.

Key words and phrases: $\beta \mathbb{N}$, Open Coloring Axiom.

The first author thanks Murray Bell and the University of Manitoba for their hospitality. 
such that $B / I$ contains $\mathcal{P}(\omega)$. The examples constructed in [Dow97, vM01] show that this is not true if $\mathrm{CH}$ is assumed. Another question suggests itself here: does it suffice to just assume that $B / I$ has an infinite completely separated family? In the third section we prove that OCA provides a positive answer to Bell's question if the space we start with has a dense subset of cardinality at most $\omega_{1}$.

The authors acknowledge the support received from the National Science Foundation (USA) via grant DMS-0103985 and NSERC, National Science Foundation (USA) grant DMS-40313-00 01, then NSERC again, respectively.

2. Extending to regular closed under OCA. Recall that OCA is the Open Coloring Axiom as formulated in $[\operatorname{Tod} 89]$. By $[X]^{2}$ we denote the reduced square of $X$, which is the set of all unordered pairs of elements of $X$. To every $Y \subseteq[X]^{2}$ naturally corresponds a symmetric subset of $X^{2}$ disjoint from the diagonal. If $X$ is a topological space then on the reduced square we consider the topology consisting of all sets that correspond to open subsets (in the product topology) of $X^{2}$. The statement OCA says:

if $X$ is separable and metrizable and if $[X]^{2}=K_{0} \cup K_{1}$, where $K_{0}$ is open, then either $X$ has an uncountable $K_{0}$-homogeneous subset $Y$ or $X$ is the union of countably many $K_{1}$-homogeneous subsets.

In Theorem 3.6 we will show that if $\mathcal{B}$ is a subalgebra of $\mathcal{P}\left(\omega_{1}\right)$ and $\mathcal{I}$ is a countably generated ideal on $\mathcal{B}$ such that $\mathcal{B} / \mathcal{I}$ contains $\mathcal{P}(\omega)$, then $\mathcal{B}$ contains $\mathcal{P}(\omega)$ in a very strong sense. Our next result applies to Boolean algebras which are not necessarily subalgebras of $\mathcal{P}\left(\omega_{1}\right)$.

Theorem 2.1 (OCA). If $B$ is a Boolean algebra and $I \subset B$ is a countably generated ideal such that $\mathcal{P}(\omega)$ embeds into the quotient algebra $B / I$, then $B$ has an infinite completely separated family.

Proof. Since $I$ is countably generated, we may choose an increasing sequence $\left\{c_{n}: n \in \omega\right\}$ of members of $I$ which generates $I$. Since $\mathcal{P}(\omega)$ embeds into $B / I$, there is an embedding of $\mathcal{P}\left(2^{<\omega}\right)$ into $B / I$ as well. For each $a \subset 2^{<\omega}$, let $\widetilde{a}$ be a member of $B$, so that $a \mapsto \widetilde{a} / I$ is an embedding of $\mathcal{P}\left(2^{<\omega}\right)$ into $B / I$. Enumerate $2^{<\omega}$ as $s_{n}(n \in \omega)$. By recursively replacing $\widetilde{\left\{s_{n}\right\}}$ with $\widetilde{\left\{s_{n}\right\}} \backslash \bigvee_{i<n} \widetilde{\left\{s_{i}\right\}}$, we may assume that for $s, t \in 2^{<\omega}$,

$$
\widetilde{\{s\}} \wedge \widetilde{\{t\}} \neq 0_{B} \text { if and only if } s=t .
$$

Assume for a moment there are infinitely many $s$ for which there is $n_{s}$ satisfying $\widetilde{\{s\}} \wedge\left(c_{m} \backslash c_{n_{s}}\right)=0_{B}$ for all $m \geq n_{s}$. Then for $b \leq \widetilde{\{s\}} \backslash c_{n_{s}}$ we 
have $b \in I$ if and only if $b=0_{B}$. And therefore $\widetilde{\{s\}} \backslash c_{n_{s}}$ form an infinite completely separated family, as witnessed by $\widetilde{a}\left(a \subseteq 2^{<\omega}\right)$.

We can therefore assume that for each $s$ and every $n$ there is $m \geq n$ such that $\widetilde{\{s\}} \wedge\left(c_{m} \backslash c_{n}\right)$ is nonzero. Find an increasing sequence $\left\{n_{i}: i \in \omega\right\} \subset \omega$ such that for every $s$ and all $i \geq|s|$ the element

$$
x(s, i)=\widetilde{\{s\}} \wedge\left(c_{n_{i+1}} \backslash c_{n_{i}}\right)
$$

is nonzero. By replacing $c_{i}$ with $c_{n_{i}}$ we may assume for simplicity that $n_{i}=i$ for all $i$, and therefore

$$
x(s, n)=\widetilde{\{s\}} \wedge\left(c_{n+|s|+1} \backslash c_{n+|s|}\right) .
$$

Then we have

(1) $x(s, n) \wedge x(t, m) \neq 0_{B}$ if and only if $(s, n)=(t, m)$.

(2) $x(s, n) \wedge c_{m}=0_{B}$ if $m \leq|s|$.

For each $f \in 2^{\omega}$, let

$$
a_{f}=\{f\lceil n: n \in \omega\} .
$$

Note that for each $s=f\left\lceil n\right.$, there is an $m$ so that $x(s, k) \leq \widetilde{a_{f}}$ for all $k \geq m$. In addition, for each $b \subset a_{f}$ and each $s \in a_{f}$, there is an $m$ so that

(3) if $s \in b$ then $x(s, k) \leq \widetilde{b}$ for all $k \geq m$,

(4) if $s \notin b$ then $x(s, k) \wedge \widetilde{b}=0_{B}$ for all $k \geq m$.

Now we will try to show that there is an $f \in 2^{\omega}$, an infinite $a \subset a_{f}$, and an $h \in \omega^{\omega}$ such that $\{x(s, h(n)): n \in a$, and $s=f\lceil n\}$ is completely separated. In actual fact, it could be proved that

$$
\left\{\widetilde{\{s\}} \backslash c_{h(n)}: n \in a \text { and } s=f\lceil n\}\right.
$$

is completely separated, but this fact will not be used.

We will use Veličković's OCA approach (as in [Vel93]). Let $\mathfrak{X}$ denote the family of all pairs $(a, b)$ of infinite sets $b \subset a \subset 2^{<\omega}$ such that there is an $f$ with $a \subset a_{f}$. Clearly then $f$ is unique and we will use the notation $f_{a}$ and $f_{b}$ to denote it. We will put a pair $\{(a, b),(c, d)\} \subset \mathfrak{X}$ into a set $K_{0}$ just in case the following three conditions hold:

(1) $f_{a} \neq f_{c}$

(2) $b \cap c=d \cap a$;

(3) there is a pair $(s, n)$ such that

(a) $x(s, n) \leq \widetilde{a} \wedge \widetilde{c}$

(b) exactly one of $\widetilde{b} \wedge x(s, n)$ and $\widetilde{d} \wedge x(s, n)$ is $0_{B}$.

Claim 1. There is no uncountable $\mathcal{Y} \subseteq \mathfrak{X}$ such that $[\mathcal{Y}]^{2} \subseteq K_{0}$. 
Proof of Claim 1. Suppose that $\mathcal{Y} \subseteq \mathfrak{X}$ is uncountable and $K_{0}$-homogeneous. Set

$$
Y=\bigcup\{b:(\exists a)(a, b) \in \mathcal{Y}\} .
$$

From the fact that $\mathcal{Y}$ is $K_{0}$-homogeneous it follows that for each $(a, b) \in \mathcal{Y}$ we have $Y \cap a=b$. Let us consider the element $\widetilde{Y}$ of $B$. For each $(a, b) \in \mathcal{Y}$, there is an $n_{(a, b)}$ such that $(\widetilde{Y} \cap \widetilde{a}) \triangle \widetilde{b}$ is contained in $c_{n_{(a, b)}}$. Fix any $n$ so that $n=n_{(a, b)}$ for an uncountable subset $\mathcal{Y}^{\prime}$ of $\mathcal{Y}$. There are only finitely many $(s, m)$ such that $x(s, m) \wedge c_{n}$ is not zero. We may assume that for each such $(s, m)$, either $\widetilde{b}$ meets $x(s, m)$ for each $(a, b) \in \mathcal{Y}^{\prime}$ or $\widetilde{b}$ is disjoint from $x(s, m)$ for each $(a, b) \in \mathcal{Y}^{\prime}$. Now select distinct $(a, b)$ and $(c, d)$ from $\mathcal{Y}^{\prime}$. Since $\{(a, b),(c, d)\} \in K_{0}$, there is a pair $(s, m)$ such that $x(s, m) \leq \widetilde{a} \wedge \widetilde{c}$ and exactly one of $\widetilde{b}$ and $\widetilde{d}$ meets $x(s, m)$. By our second reduction of $\mathcal{Y}^{\prime}$ it follows that $x(s, m)$ is disjoint from $c_{n}$. Since $(\widetilde{Y} \wedge \widetilde{a}) \backslash c_{n}=\widetilde{b} \backslash c_{n}$, we have $\widetilde{Y} \wedge x(s, m)=\widetilde{b} \wedge x(s, m)$, and since $(\widetilde{Y} \wedge \widetilde{c}) \backslash c_{n}=\widetilde{d} \backslash c_{n}$, we have $\widetilde{Y} \wedge x(s, m)=\widetilde{d} \wedge x(s, m)$, a contradiction.

Consider the following topology on $\mathfrak{X}$. An open set $[\varphi]$ is obtained by specifying a function, $\varphi$, from a finite set $F \subset 2 \times 2^{<\omega} \times \omega$ into 2 . Given such a $\varphi$ with domain $F$, a pair $(a, b)$ is a member of $[\varphi]$ if the following hold:

(i) for each $(0, s, 0) \in F, s \in a$ if and only if $\varphi(0, s, 0)=1$,

(ii) for each $(1, s, 0) \in F, s \in b$ if and only if $\varphi(1, s, 0)=1$,

(iii) for each $(0, s, n) \in F$ with $n \geq 1, x(s, n) \leq \widetilde{a}$ if and only if $\varphi(0, s, n)=1$,

(iv) for each $(1, s, n) \in F$ with $n \geq 1,0 \neq \widetilde{b} \wedge x(s, n)$ if and only if $\varphi(1, s, n)=1$.

This results in a separable metric topology on $\mathcal{X}$ and we may note that $K_{0}$ is an open subset of the square.

Claim 2. If there are $\mathcal{Y}_{n}(n \in \omega)$ such that $\mathfrak{X}=\bigcup_{n} \mathcal{Y}_{n}$ and $\left[\mathcal{Y}_{n}\right]^{2} \cap K_{0}$ $=\emptyset$ for all $n$, then there is an infinite completely separated subset of $B$.

Proof of Claim 2. Assume the contrary, i.e. that $B$ contains no infinite completely separated sequence. For each $k$, let $Y_{k}$ be a countable dense subset of $\mathcal{Y}_{k}$ with respect to the above-mentioned topology. Fix any $g \in 2^{\omega}$ such that $g \neq f_{a}$ for all $(a, b) \in \bigcup_{k} Y_{k}$. For each $n \in \omega$, let $s_{n}=g\lceil n$.

Recursively construct an increasing sequence $\left\langle n_{i}: i \in \omega\right\rangle$. Let $n_{0}=0$ and suppose that $\left\langle n_{i}: i \leq k\right\rangle$ has been defined. For each $i \leq k$ and each $b^{\prime} \subset a^{\prime} \subset$ $\left\{s_{j}: j<n_{k}\right\}$, fix, if possible, a pair $(a, b) \in Y_{i}$ such that $a^{\prime}=a \cap\left\{s_{j}: j<n_{k}\right\}$ and $b^{\prime}=b \cap\left\{s_{j}: j<n_{k}\right\}$. Thus, we have chosen some finite set of pairs from $\bigcup\left\{Y_{i}: i \leq k\right\}$. Fix $n_{k+1}$ large enough so that $g\left\lceil n_{k+1} \neq f_{a}\left\lceil n_{k+1}\right.\right.$ for each $a$ from one of these finitely many pairs. 
Set $A=\bigcup\left\{\left[n_{k}, n_{k+1}\right): k\right.$ is even $\}$ and $a=\left\{s_{j}: j \in A\right\}$. Fix any function $h_{0} \in \omega^{\omega}$ such that for each $n \in A$ and each $m>h_{0}(n)$ we have $x\left(s_{n}, m\right) \leq \widetilde{a}$.

For each $h \geq h_{0}$, fix an infinite $J_{h} \subset A$ such that $A_{h}=\left\{x\left(s_{n}, h(n)\right)\right.$ : $\left.n \in J_{h}\right\}$ is not separated from $C_{h}=\left\{x\left(s_{n}, h(n)\right): n \in A \backslash J_{h}\right\}$. Now temporarily set $b_{h}=\left\{s_{n}: n \in J_{h}\right\}$ and consider $\widetilde{b_{h}}$. It follows that at least one of $\widetilde{b_{h}}$ and $\widetilde{a} \backslash \widetilde{b_{h}}$ meets an infinite subset of each of $A_{h}$ and $C_{h}$. If it is the former, then leave $b_{h}$ as it is, if the latter, then reassign $b_{h}$ to $a \backslash b_{h}$. In either case, it follows that the set

$$
z_{h}=\left\{s \in a \backslash b_{h}: \widetilde{b_{h}} \wedge x(s, h(|s|)) \neq 0_{B}\right\}
$$

is infinite.

For each $h$, there is an $i$ such that $\left(a, b_{h}\right)$ is a member of $\mathcal{Y}_{i}$. Therefore there is an $i$ such that the family of strictly increasing $h$ for which $\left(a, b_{h}\right) \in \mathcal{Y}_{i}$ is dominating modulo finite in $\omega^{\omega}$.

Claim 3. There is an $m$ such that for each $l$, there is an $h$ such that $\left(a, b_{h}\right) \in \mathcal{Y}_{i}, h(m) \geq l$, and $s_{m} \in z_{h}$.

Proof of Claim 3. Otherwise, we can, for each $m$, define $f(m)$ so that for $h$ with $\left(a, b_{h}\right) \in \mathcal{Y}_{i}$, either $s_{m} \notin z_{h}$ or $h(m)<f(m)$. Having defined such an $f$, choose $h$ with $\left(a, b_{h}\right) \in \mathcal{Y}_{i}$ so that there is an $m^{\prime}$ such that $f(m)<h(m)$ for all $m \geq m^{\prime}$. Since $z_{h}$ is infinite, there is an $m \geq m^{\prime}$ such that $s_{m} \in z_{h}$. Obviously this contradicts the choice of $f$.

Fix any $m$ as in Claim 3. Choose a family $\left\{h_{l}: l \in \omega\right\}$ such that, for each $l, s_{m} \in z_{h_{l}}, h_{l}(m) \geq l$, and $\left(a, b_{h_{l}}\right) \in \mathcal{Y}_{i}$. (We shall write $b_{l}$ for $b_{h_{l}}$ from now on.) By passing to a subsequence and re-enumerating, we can assume that $b_{k} \cap\left\{s_{j}: j \leq l\right\}=b_{l} \cap\left\{s_{j}: j \leq l\right\}$ for each $k \geq l$.

Fix the minimal odd $k$ such that $m<n_{k}$. By the density of $Y_{i}$ and the choice of the $n_{k}$ 's, there is a pair $(c, d) \in Y_{i}$ such that $c \cap\left\{s_{j}: j<n_{k}\right\}$ $=a \cap\left\{s_{j}: j<n_{k}\right\}, d \cap\left\{s_{j}: j<n_{k}\right\}=b_{n_{k}} \cap\left\{s_{j}: j<n_{k}\right\}$ and $f_{c}\left\lceil n_{k+1} \neq\right.$ $g\left\lceil n_{k+1}\right.$. Of course, since $k$ is odd, $A \cap\left[n_{k}, n_{k+1}\right)$ is empty, and since $c \subset a_{f_{c}}$, $c \cap\left\{s_{j}: n_{k+1} \leq j\right\}$ is empty. Therefore,

$$
a \cap d=c \cap b_{l} \quad \text { for all } l \geq n_{k} .
$$

Finally, note that $s_{m} \in c \backslash d$ since $s_{m} \in z_{h_{n_{k}}} \subset a \backslash b_{n_{k}}$. Therefore, there is an $l$ such that

$$
x\left(s_{m}, j\right) \leq \widetilde{c} \backslash \widetilde{d}, \quad x\left(s_{m}, j\right) \leq \widetilde{a} \quad \text { for all } j \geq l .
$$

So we have $x\left(s_{m}, h_{l}(m)\right) \wedge \widetilde{b}_{l} \neq 0_{B}$ and $x\left(s_{m}, h_{l}(m)\right) \wedge \widetilde{d}=0_{B}$. By this, $(*)$, $(* *)$ and $f_{a} \neq f_{c}$, we have $\left\{\left(a, b_{l}\right),(c, d)\right\} \in K_{0}$, a contradiction. Claim 2 is proved.

This completes the proof of Theorem 2.1. 
3. Completely additive almost liftings. If $h: Y \rightarrow X$ is any partial function, then define $\Phi_{h}: \mathcal{P}(X) \rightarrow \mathcal{P}(Y)$ by

$$
\Phi_{h}(A)=h^{-1}(A) .
$$

A mapping $F: \mathcal{P}(X) \rightarrow \mathcal{P}(Y)$ is completely additive if there is an $h: Y \rightarrow X$ such that $F=\Phi_{h}$.

If $\theta$ is a cardinal then an ideal $\mathcal{J}$ on $\omega$ is $\theta$-cc over fin if every family of $\mathcal{J}$-positive subsets of $\omega$ that are pairwise almost disjoint modulo finite has size less than $\theta$. If $\theta=\aleph_{1}$ then we say that $\mathcal{J}$ is $c c c$ over fin (see [Far00, $\S 3.3])$.

If $X$ is a set, $\mathcal{J}$ is an ideal on $\omega, \mathcal{I}$ is an ideal on $X$, and $\Phi: \mathcal{P}(\omega) / \mathcal{J} \rightarrow$ $\mathcal{P}(X) / \mathcal{I}$ is a Boolean algebra homomorphism, then a map $\Phi_{*}: \mathcal{P}(\omega) \rightarrow \mathcal{P}(X)$ is a lifting of $\Phi$ if the diagram

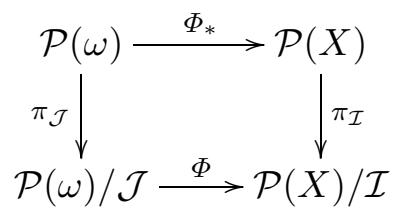

commutes, or in other words, if the formula $\left[\Phi_{*}(A)\right]_{\mathcal{I}}=\Phi\left([A]_{\mathcal{J}}\right)$ is true for all $A \in \mathcal{P}(\omega)$. In the case that $\Phi: \mathcal{P}(\omega) \rightarrow \mathcal{P}(X) / \mathcal{I}$ is a homomorphism, a lifting of $\Phi$ is a function $\Phi_{*}: \mathcal{P}(\omega) \rightarrow \mathcal{P}(X)$ such that $\left[\Phi_{*}(A)\right]_{\mathcal{I}}=\Phi(A)$ is true for all $A \in \mathcal{P}(\omega)$. Since we are not requiring the lifting to have any algebraic properties, the Axiom of Choice implies that every homomorphism has a lifting. The symbol $\Phi_{*}$ will always stand for a lifting of $\Phi$. A map $F: \mathcal{P}(\omega) \rightarrow \mathcal{P}(X)$ is an almost lifting if the family

$$
\left\{A:[F(A)]_{\mathcal{I}}=\Phi\left([A]_{\mathcal{J}}\right)\right\}
$$

includes an ideal that is ccc over fin. Again, in the case that there is no $\mathcal{J}$ involved, $F$ is an almost lifting if the family $\left\{A:[F(A)]_{\mathcal{I}}=\Phi(A)\right\}$ includes an ideal that is ccc over fin.

If $\Phi: \mathcal{P}(\omega) \rightarrow \mathcal{P}(X) / \mathcal{I}$ is a homomorphism and $B \subseteq X$ then let

$$
\Phi^{B}: \mathcal{P}(\omega) \rightarrow \mathcal{P}(B) / \mathcal{I}
$$

be the homomorphism whose lifting is $C \mapsto \Phi_{*}(C) \cap B$, which we denote as $\Phi_{*}^{B}$.

Lemma 3.1. Assume $\mathcal{I}$ is an analytic ideal and $\Phi: \mathcal{P}(\omega) /$ fin $\rightarrow \mathcal{P}(\omega) / \mathcal{I}$ is a homomorphism. The following are equivalent:

(1) $\Phi$ has a continuous almost lifting.

(2) There is $B \subseteq \omega$ such that $\Phi^{B}$ has a continuous lifting and $\operatorname{ker}\left(\Phi^{\omega \backslash B}\right)$ is ccc over fin. 
Proof. This is [Far00, Lemma 3.3.4], using the fact that a homomorphism has a Baire-measurable lifting if and only if it has a continuous lifting (see [Far00, Lemma 1.3.2]).

TheOREM 3.2. Assume $O C A$ and $M A$ and let $\mathcal{I}$ be a countably generated ideal on $\omega$. Then every homomorphism $\Phi: \mathcal{P}(\omega) /$ fin $\rightarrow \mathcal{P}(\omega) / \mathcal{I}$ has a completely additive almost lifting.

Proof. By [Far00, Theorem 3.3.6], $\Phi$ has a continuous almost lifting. By the second part of the same result, there is $A \subseteq \omega$ such that $\Phi^{A}$ has a continuous lifting and $\operatorname{ker}\left(\Phi^{\omega \backslash A}\right)$ is ccc over fin. Since the restriction of $\mathcal{I}$ to $A$ is countably generated, it is Rudin-Keisler isomorphic (see [Far00]) to either fin or fin $\times \emptyset$. By [Far00, Theorems 1.6.1 and 1.6.2], $\Phi^{A}$ has a completely additive lifting $\Phi_{h}$, and this is a completely additive almost lifting for $\Phi$.

We will need the following improvement of Theorem 3.2 (or rather its consequence, Corollary 3.4) that does not require $\operatorname{ker}(\Phi) \supseteq$ fin. The proof of Theorem 3.2 can be modified to a proof of Corollary 3.4 by making only minor changes. For the convenience of the reader, we will give another proof, using only those results that were both stated and proved in [Far00], instead of their proofs.

THEOREM 3.3. Assume that for every countably generated ideal $\mathcal{I}$ on $\omega$ every homomorphism from $\mathcal{P}(\omega) /$ fin into $\mathcal{P}(\omega) / \mathcal{I}$ has a completely additive almost lifting. Then for every countably generated ideal $\mathcal{I}$ on $\omega$ every homomorphism of $\mathcal{P}(\omega)$ into $\mathcal{P}(\omega) / \mathcal{I}$ has a completely additive almost lifting.

Proof. Let $\Phi: \mathcal{P}(\omega) \rightarrow \mathcal{P}(\omega) / \mathcal{I}$ be a homomorphism, and let $\Phi_{*}$ be its lifting. For $n \in \omega$ let

$$
A_{n}=\Phi_{*}(\{n\})
$$

We first show that we may assume that no $A_{n}$ is in $\mathcal{I}$ and that fin $\subset \mathcal{I}$. Let $S_{0}=\{n:\{n\} \in \operatorname{ker}(\Phi)\}$ and $S_{1}=\omega \backslash S_{0}$.

We can define $\Phi_{0}: \mathcal{P}\left(S_{0}\right) \rightarrow \mathcal{P}\left(\Phi_{*}\left(S_{0}\right)\right) / \mathcal{I}$ and $\Phi_{1}: \mathcal{P}\left(S_{1}\right) \rightarrow \mathcal{P}\left(\Phi_{*}\left(S_{1}\right)\right) / \mathcal{I}$ as the restrictions of $\Phi$. By our assumption, $\Phi_{0}$ has a completely additive almost lifting, given by say $h_{0}$, and if we are able to find $h_{1}$ for $\Phi_{1}$, then we can define $h$ to simply be $h_{0} \cup h_{1}$ and $\Phi_{h}$ is easily seen to be a completely additive almost lifting of $\Phi$. Therefore we may assume that $\operatorname{ker}(\Phi)$ is $\{\emptyset\}$.

Now let $D=\omega \backslash \bigcup \mathcal{I}$. For each $d \in D$, since $\Phi$ is a homomorphism and $\Phi_{*}$ is a lifting,

$$
\mathcal{U}_{d}=\left\{C: d \in \Phi_{*}(C)\right\}
$$

is an ultrafilter on $\omega$. For each $d \in D$ such that $\mathcal{U}_{d}$ is a fixed ultrafilter, $d$ will be in the domain of $h_{D}$ and define $h_{D}(d)$ to be that integer. Therefore, for 
$C \subset \omega, \Phi_{h_{D}}(C)$ will equal $\Phi_{*}(C) \cap \operatorname{dom}\left(h_{D}\right)$. For any $C \subset \omega$ such that $C \notin \mathcal{U}_{d}$ for all $d \in D \backslash \operatorname{dom}\left(h_{D}\right)$, we have $\Phi_{h_{D}}(C)=\Phi_{*}(C) \cap D$. The ideal generated by such $C$ is ccc over fin. That is, $\Phi^{D}$ has a completely additive almost lifting, hence it suffices to prove that $\Phi^{\omega \backslash D}$ also has such a lifting.

Therefore, we may also assume that $\bigcup \mathcal{I}=\omega$. We now let $\mathcal{I}_{1}$ be the ideal generated by $\mathcal{I}$ and $\left\{A_{n}: n \in \omega\right\}$. By replacing $A_{n}$ with $\left(\{n\} \cup A_{n}\right) \backslash$ $\left(\bigcup_{i<n} A_{i} \cup n\right)$, we may assume that $\omega$ is a disjoint union of $A_{n}(n \in \omega)$. Then $\Phi_{*}$ is a lifting of a homomorphism $\Psi$ of $\mathcal{P}(\omega)$ into $\mathcal{P}(\omega) / \mathcal{I}_{1}$ such that $\operatorname{ker}(\Psi) \supseteq$ fin. Since $\mathcal{I}_{1}$ is countably generated, by our assumption there is a partial function $h: \omega \rightarrow \omega$ such that $\Phi_{h}$ is a completely additive almost lifting of $\Psi$. Let $\mathcal{J}$ be a ccc over fin ideal such that $\Phi_{h}$ is a lifting of $\Psi$ on $\mathcal{J}$.

CASE 1. Assume there is $\bar{m}$ such that for all $n \geq \bar{m}$ we have (writing $A={ }_{\mathcal{I}} B$ for $\left.A \triangle B \in \mathcal{I}\right)$

$$
A_{n}={ }_{\mathcal{I}} h^{-1}(\{n\}) .
$$

Define a partial function $h_{1}: \omega \rightarrow \omega$ so that

- $h_{1}$ agrees with $h$ on $\omega \backslash \bigcup_{i<\bar{m}} A_{i}$,

- $h_{1}(k)=i$ if $k \in A_{i}$ and $i<\bar{m}$.

We claim that $\Phi_{h_{1}}$ is an almost lifting for $\Phi$. It will suffice to check that for $X \in \mathcal{J}$ we have

$$
D_{X}=\Phi_{h_{1}}(X) \triangle \Phi_{*}(X) \in \mathcal{I} .
$$

Since $\Phi_{h}(X)=\mathcal{I}_{1} \Phi_{*}(X)$, there is $n \geq \bar{m}$ such that $\Phi_{h}(X)==_{\mathcal{I}} \Phi_{*}(X) \backslash$ $\bigcup_{i<n} A_{i}$. Write $\bar{A}=\bigcup_{i<n} A_{i}$. We have $\Phi_{h_{1}}(X) \triangle \Phi_{h}(X) \subseteq \bar{A}$, therefore $D_{X} \backslash \bar{A} \in \mathcal{I}$. But $\Phi_{h_{1}}(X) \cap \bar{A}$ is equal to $\bigcup_{i \in X \cap n} A_{i}$ modulo $\mathcal{I}$, which in turn is equal to $\Phi_{*}(X) \cap \bar{A}$ modulo $\mathcal{I}$. Therefore $D_{X} \cap \bar{A} \in \mathcal{I}$, and this concludes the proof in Case 1 .

CAse 2. The set $X_{0}=\left\{n: A_{n} \triangle h^{-1}(\{n\}) \notin \mathcal{I}\right\}$ is infinite. Note that for every $Y \subseteq \omega$ and all $i$ we have

- $i \in Y$ if and only if $A_{i} \backslash \Phi_{*}(Y) \in \mathcal{I}$,

- $i \notin Y$ if and only if $A_{i} \cap \Phi_{*}(Y) \in \mathcal{I}$.

If moreover $Y \in \mathcal{J}$ then we have $\Phi_{h}(Y)={ }_{\mathcal{I}_{1}} \Phi_{*}(Y)$ and therefore

(*) for all but finitely many $i$ we have $i \in Y$ if and only if $A_{i} \backslash \Phi_{h}(Y) \in \mathcal{I}$, (**) for all but finitely many $i$ we have $i \notin Y$ if and only if $A_{i} \cap \Phi_{h}(Y) \in \mathcal{I}$.

We will find $Y \in \mathcal{J}$ that contradicts $(*)$ or $(* *)$, therefore proving that Case 2 leads to contradiction.

Let us first consider the subcase when

$$
X_{1}=\left\{i \in X_{0}:\left(\exists n_{i} \neq i\right) h^{-1}\left(\left\{n_{i}\right\}\right) \cap A_{i} \notin \mathcal{I}\right\}
$$


is infinite. First find an infinite $X_{2} \subseteq X_{1}$ such that

$$
X_{2} \cap\left\{n_{i}: i \in X_{2}\right\}=\emptyset .
$$

Then find $Y \subseteq\left\{n_{i}: i \in X_{2}\right\}$ such that $Y \in \mathcal{J}$ and $\left\{i \in X_{2}: n_{i} \in Y\right\}$ is infinite (we are allowing $Y$ itself to be finite). But there are infinitely many $i \in X_{2}$ such that $n_{i} \in Y$. Therefore $\Phi_{h}(Y) \cap A_{i} \notin \mathcal{I}$ for all such $i$, contradicting $(* *)$.

So it only remains to check the case when for all but finitely many $i \in X_{0}$ we have

$$
h^{-1}(\{n\}) \cap A_{i} \in \mathcal{I}
$$

for all $n \neq i$. Let $X_{1}$ be the set of such $i$. Since $\mathcal{I}$ is countably generated, for each $i \in X_{1}$ we can find an infinite $J_{i} \subseteq \omega$ such that

$$
\text { for every infinite } C \subseteq J_{i} \text { we have } \Phi_{h}(C) \cap A_{i} \notin \mathcal{I} \text {. }
$$

Find an infinite $Y \subseteq X_{1}$ such that $Y \in \mathcal{J}$ and $J_{i} \backslash Y$ is infinite for all $i \in X_{1}$. Then for every $i \in Y$ we have $A_{i} \backslash \Phi_{h}(Y) \notin \mathcal{I}$, contradicting (*).

This exhausts all possibilities and concludes the proof.

The following is an immediate consequence of Theorems 3.2 and 3.3.

Corollary 3.4. Assume $O C A$ and $M A$ and let $\mathcal{I}$ be a countably generated ideal on $\omega$. Then every homomorphism of $\mathcal{P}(\omega)$ into $\mathcal{P}(\omega) / \mathcal{I}$ has a completely additive almost lifting.

Definition 3.5. If $\theta$ is a cardinal, $\lambda$ is any set, and $\Phi: \mathcal{P}(\omega) / \mathcal{J} \rightarrow$ $\mathcal{P}(\lambda) / \mathcal{I}$ is a homomorphism, then $F: \mathcal{P}(\omega) \rightarrow \mathcal{P}(\lambda)$ is a $\theta$-almost lifting of $\Phi$ if the set

$$
\left\{A \in \mathcal{P}(\omega): \Phi_{*}(A)=_{\mathcal{I}} F(A)\right\}
$$

includes an ideal that is $\theta$-cc over fin.

In particular, an $\aleph_{1}$-almost lifting is an almost lifting in the usual sense. The main result of this section is the following.

TheOREm 3.6. Assume $O C A$ and $M A$ and let $\mathcal{I}$ be a countably generated ideal on $\omega_{1}$. Then every homomorphism $\Phi: \mathcal{P}(\omega) \rightarrow \mathcal{P}\left(\omega_{1}\right) / \mathcal{I}$ has a completely additive $\aleph_{2}$-almost lifting.

Proof. Let $\Phi$ be as above and let $\Phi_{*}$ be a lifting for $\Phi$. Fix an increasing sequence $A_{n}(n \in \omega)$ of subsets of $\omega_{1}$ which generate $\mathcal{I}$. Let $D=\omega_{1} \backslash$ $\bigcup_{n \in \omega} A_{n}$. Then, analogously to what we saw in Theorem $3.3, \Phi^{D}$ has a completely additive lifting. Also, if $\bigcup_{n \in \omega} A_{n}$ is countable, then $\Phi$ has a completely additive almost lifting by Theorem 3.2. Hence we may assume

$$
\omega_{1}=\bigcup_{n \in \omega} A_{n} .
$$


Let $\mathcal{J}$ be the ideal orthogonal to $\mathcal{I}$ :

$$
\mathcal{J}=\left\{B: A_{n} \cap B \text { is finite for all } n\right\} .
$$

By our convention that $\omega_{1}$ is covered by the union of $A_{n}$ 's, every set in $\mathcal{J}$ is countable. An easy diagonalization argument shows that if $B_{n} \in \mathcal{J}$ for $n \in \omega$, then there is $B \in \mathcal{J}$ such that $B_{n} \backslash B$ is finite for all $n$. (Such a family $\mathcal{J}$ is usually called a $P$-ideal of countable sets, or just a P-ideal if $\mathcal{J} \subseteq \mathcal{P}(\omega)$.)

By Corollary 3.4, for every $B \in\left[\omega_{1}\right]^{\aleph_{0}}$ we can fix $\bar{B} \subseteq B$ and $h_{B}: \bar{B} \rightarrow \omega$ such that the mapping $\Phi_{h_{B}}$ is an almost lifting of $\Phi^{\bar{B}}$. Define partitions $\left[\left[\omega_{1}\right]^{\aleph_{0}}\right]^{2}=K_{0} \cup K_{1},\left[\left[\omega_{1}\right]^{\aleph_{0}}\right]^{2}=K_{0}^{n} \cup K_{1}^{n}(n \in \omega)$, as follows:

(1) $\{A, B\} \in K_{0}$ if and only if $h_{A}(\xi) \neq h_{B}(\xi)$ for some $\xi \in A \cap B$,

(2) $\{A, B\} \in K_{0}^{n}$ if and only if $h_{A}(\xi) \neq h_{B}(\xi)$ for some $\xi \in(A \cap B) \backslash A_{n}$.

The following is almost identical to [Far00, Lemma 3.8.4], but we will reproduce the proof for the reader's convenience.

Lemma 3.7. For all $A, B$ in $\left[\omega_{1}\right]^{\aleph_{0}}$ there is a large enough $n$ such that $\{A, B\} \in K_{1}^{n}$.

Proof. Assume there is no such $n$. For every $n$ find $\xi_{n} \in(A \cap B) \backslash A_{n}$ such that $h_{A}\left(\xi_{n}\right) \neq h_{B}\left(\xi_{n}\right)$. By applying Ramsey's theorem, we may find an infinite $D \subseteq \omega$ such that $h_{A}\left(\xi_{m}\right) \neq h_{B}\left(\xi_{n}\right)$ for all $\{m, n\} \subseteq D$. The family $\left(\left(\Phi^{A}\right)_{*}\right.$ and $\left(\Phi^{B}\right)_{*}$ are arbitrary liftings of $\Phi^{A}$ and $\Phi^{B}$, respectively)

$$
\left\{C \subseteq \omega:\left(\Phi^{A}\right)_{*}(C)=_{\mathcal{I}} \Phi_{h_{A}}(C) \&\left(\Phi^{B}\right)_{*}(C)=_{\mathcal{I}} \Phi_{h_{B}}(C)\right\}
$$

includes an ideal that is ccc over fin, and therefore nonmeager ([Far00, Lemma 3.3.2]). So we can find an infinite $C \subseteq \omega$ such that the set

$$
\bigcup_{n \in C}\left\{h_{A}\left(\xi_{n}\right), h_{B}\left(\xi_{n}\right)\right\}
$$

belongs to this ideal (by a well known result of Jalali-Naini and Talagrand, see e.g. [Far00, Lemma 3.10.2]). The sets

$$
X_{A}=\left\{h_{A}\left(\xi_{n}\right): n \in C\right\}, \quad X_{B}=\left\{h_{B}\left(\xi_{n}\right): n \in C\right\}
$$

are disjoint. Hence $\Phi_{*}\left(X_{A}\right) \cap \Phi_{*}\left(X_{B}\right) \in \mathcal{I}$. But $\Phi_{*}\left(X_{A}\right) \cap A$ is equal to $h_{A}^{-1}\left(X_{A}\right)$ modulo $\mathcal{I}$, and $\Phi_{*}\left(X_{B}\right) \cap B$ is equal to $h_{B}^{-1}\left(X_{B}\right)$ modulo $\mathcal{I}$. Since $h_{A}^{-1}\left(X_{A}\right) \cap h_{B}^{-1}\left(X_{B}\right)$ is not included in any $A_{n}$ (as it contains $\xi_{m}$ for some $m>n)$, we have a contradiction.

Consider $\left[\omega_{1}\right]^{\aleph_{0}}$ and $\mathcal{J}$ as posets under the ordering $\subseteq^{*}$ of inclusion modulo finite: $A \subseteq^{*} B$ if and only if $A \backslash B$ is finite. Since $\left[\omega_{1}\right]^{\aleph_{0}}$ and $\mathcal{J}$ are P-ideals of countable sets, the posets $\left(\left[\omega_{1}\right]^{\aleph_{0}}, \subseteq^{*}\right)$ and $\left(\mathcal{J}, \subseteq^{*}\right)$ are $\sigma$-directed. 
LEMMA 3.8. If for some $\bar{m}$ there is $\mathcal{X}_{1} \subseteq\left[\omega_{1}\right]^{\aleph_{0}}$ that is $K_{1}^{\bar{m}}$-homogeneous and cofinal in $\left(\left[\omega_{1}\right]^{\aleph_{0}}, \subseteq^{*}\right)$, then $\Phi$ has a completely additive $\aleph_{2}$-almost lifting.

Proof. The homogeneity of $\mathcal{X}_{1}$ implies that

$$
h=\bigcup_{B \in \mathcal{X}_{1}} h_{B}\left\lceil\left(B \backslash A_{\bar{m}}\right)\right.
$$

is a function. Assume $\Phi_{h}$ is not an $\aleph_{2}$-almost lifting of $\Phi$, and let $B_{\xi}\left(\xi<\omega_{2}\right)$ be an almost disjoint family of subsets of $\omega$ such that the set

$$
Y_{\xi}=\Phi_{*}\left(B_{\xi}\right) \triangle \Phi_{h}\left(B_{\xi}\right)
$$

is not in $\mathcal{I}$ for all $\xi$. Since $\mathcal{I}$ is countably generated, for every $X \in \mathcal{I}^{+}$there is a $\delta<\omega_{1}$ such that $X \cap \delta \in \mathcal{I}^{+}$. Find a countable ordinal $\delta$ such that $Y_{\xi} \cap \delta$ is not in $\mathcal{I}$ for $\aleph_{2}$ many $\xi$. There is $B \in \mathcal{X}_{1}$ such that $\delta \subseteq \subseteq^{*} B$. Since $\Phi_{h_{B}}\left(B_{\xi}\right) \backslash A_{\bar{m}}=\Phi_{h}\left(B_{\xi}\right) \cap\left(B \backslash A_{\bar{m}}\right)$ and

$$
\begin{aligned}
{\left[\left(\Phi_{*}\left(B_{\xi}\right) \cap B\right) \triangle \Phi_{h_{B}}\left(B_{\xi}\right)\right] \backslash A_{\bar{m}} } & =\left[\left(\Phi_{*}\left(B_{\xi}\right) \cap B\right) \triangle \Phi_{h}\left(B_{\xi}\right) \cap B\right] \backslash A_{\bar{m}} \\
& =\left[\Phi_{*}\left(B_{\xi}\right) \triangle \Phi_{h}\left(B_{\xi}\right)\right] \cap\left[B \backslash A_{\bar{m}}\right] \\
& =Y_{\xi} \cap\left[B \backslash A_{\bar{m}}\right],
\end{aligned}
$$

we have $\left(\Phi_{*}\left(B_{\xi}\right) \cap B\right) \triangle \Phi_{h_{B}}\left(B_{\xi}\right) \notin \mathcal{I}$ for uncountably many $\xi$, contradicting the fact that $\Phi_{h_{B}}$ is an almost lifting of $\Phi^{B}$.

In order to prove the assumption of Lemma 3.8 we will define partitions $\left[\left[\omega_{1}\right]^{\aleph_{0}}\right]^{2}=L_{0}(C) \cup L_{1}(C)(C \subseteq \omega)$ and $\left[\left[\omega_{1}\right]^{\aleph_{0}}\right]^{2}=L_{0}^{n}(C) \cup L_{1}^{n}(C)(n \in \omega$, $C \subseteq \omega)$ as follows:

(1) $\{A, B\} \in L_{1}(C)$ if and only if $h_{A}(\xi) \in C \Leftrightarrow h_{B}(\xi) \in C$ for all $\xi \in A \cap B$

(2) $\{A, B\} \in L_{1}^{n}(C)$ if and only if $h_{A}(\xi) \in C \Leftrightarrow h_{B}(\xi) \in C$ for all $\xi \in(A \cap B) \backslash A_{n}$.

LEMMA 3.9. For every $C \subseteq \omega$ the following hold:

(a) There are $\mathcal{H}_{n}(n \in \omega)$ such that each $\mathcal{H}_{n}$ is $L_{1}^{n}(C)$-homogeneous and $\left[\omega_{1}\right]^{\aleph_{0}}=\bigcup_{n} \mathcal{H}_{n}$.

(b) There are $\mathcal{H}_{n}^{\prime}(n \in \omega)$ such that each $\mathcal{H}_{n}^{\prime}$ is $L_{1}^{n}(C)$-homogeneous and $\mathcal{J}=\bigcup_{n} \mathcal{H}_{n}^{\prime}$

(c) $\mathcal{J}$ has no uncountable $L_{0}^{k}(C)$-homogeneous subsets for any $k$.

Proof. (a) For each $B \in\left[\omega_{1}\right]^{\aleph_{0}}$, since $B$ is countable it follows from [Far00, Lemma 3.3.4] that there is a $\bar{B} \subseteq B$ such that $\Phi_{h_{B}}$ is a lifting of $\Phi^{\bar{B}}$ and $\operatorname{ker}\left(\Phi^{B \backslash \bar{B}}\right)$ is ccc over fin. We may assume that $\bar{B}=\operatorname{dom}\left(h_{B}\right)$. There is also an $n=n(B)$ such that

$$
\Phi_{h_{B}}(C) \triangle\left(\Phi_{*}(C) \cap \bar{B}\right) \subseteq A_{n} .
$$

Let

$$
\mathcal{H}_{n}=\left\{B \in\left[\omega_{1}\right]^{\aleph_{0}}: n(B)=n\right\}
$$


It will suffice to show that each $\mathcal{H}_{n}$ is $L_{1}^{n}(C)$-homogeneous. Fix $A$ and $B$ in $\mathcal{H}_{n}$. Pick $\xi \in\left(\operatorname{dom}\left(h_{A}\right) \cap \operatorname{dom}\left(h_{B}\right)\right) \backslash A_{n}$. To check that $\{A, B\} \in L_{1}^{n}(C)$, note that $h_{B}(\xi) \in C$ if and only if $\xi \in \Phi_{*}(C)$ if and only if $h_{A}(\xi) \in C$. Hence $\{A, B\} \in L_{1}^{n}(C)$.

Clause (b) follows from (a) since $\mathcal{J} \subset\left[\omega_{1}\right]^{\aleph_{0}}$.

In order to prove (c), we may assume $k=0$ since $L_{0}^{k}(C) \subseteq L_{0}^{0}(C)=$ $L_{0}(C)$ for all $k$. Fix $\mathcal{Y}=\left\{B_{\alpha}: \alpha<\omega_{1}\right\}$ included in $\mathcal{J}$. By part (b), it has an uncountable intersection with some $\mathcal{H}_{n}^{\prime}$, so we may assume $\mathcal{Y}$ is $L_{1}^{n}(C)$-homogeneous. By refining, we may assume that the sets

$$
F_{\alpha}=B_{\alpha} \cap A_{n}
$$

form a $\Delta$-system with root $R$, and that the functions $\chi_{C} \circ h_{B_{\alpha}}$ agree on $R\left(\chi_{C}\right.$ is the characteristic function of $C)$. For $\alpha<\beta$ pick $\xi \in B_{\alpha} \cap B_{\beta}=\bar{B}_{\alpha} \cap \bar{B}_{\beta}$. We claim that

$$
\chi_{C} \circ h_{B_{\alpha}}(\xi)=\chi_{C} \circ h_{B_{\beta}}(\xi) .
$$

If $\xi \notin A_{n}$, this follows by the $L_{1}^{n}(C)$-homogeneity. If $\xi \in A_{n}$, then $\xi \in R$, hence the conclusion follows. We have shown that every uncountable subset of $\left[\omega_{1}\right]^{\aleph_{0}}$ contains an uncountable $L_{1}(C)$-homogeneous subset, and this implies (c).

Let $\mathcal{C}$ be the forcing for adding a single Cohen subset of $\omega$, and let $\dot{C}$ be the canonical $\mathcal{C}$-name for it. Since $\mathcal{C}$ has the countable chain condition, it forces that $\left(\mathcal{J}^{V}, \subseteq^{*}\right)$ and $\left(\left(\left[\omega_{1}\right]^{\aleph_{0}}\right)^{V}, \subseteq^{*}\right)$ are still $\sigma$-directed in the extension. For $B \in\left(\left[\omega_{1}\right]^{\aleph_{0}}\right)^{V}$ let $h_{B}^{\dot{C}}=\chi_{\dot{C}} \circ h_{B}\left(\chi_{\dot{C}}\right.$ is the characteristic function of $\left.\dot{C}\right)$.

Assume for a moment that some condition $p \in \mathcal{C}$ forces that there is $\bar{n}$ for which some $L_{1}^{\bar{n}}(\dot{C})$-homogeneous set $\dot{\mathcal{H}}$ is cofinal in $\left(\left(\left[\omega_{1}\right]^{\aleph_{0}}\right)^{V}, \subseteq^{*}\right)$. (It should be noted that this does not follow by Lemma 3.9, in particular because $\Phi_{*}(\dot{C})$ is not defined.) Since $\mathcal{C}$ is countable, there is a single condition $q \leq p$ such that

$$
\mathcal{X}=\{B: q \Vdash \check{B} \in \dot{\mathcal{H}}\}
$$

is cofinal in $\left(\left[\omega_{1}\right]^{\aleph_{0}}, \subseteq^{*}\right)$. For $B \in \mathcal{X}$, fix $m_{B}$ such that

(1) $B \cap\left(\Phi_{h_{B}}(s) \triangle \Phi_{*}(s)\right) \subseteq A_{m(B)}$ for all $s \subseteq \operatorname{dom}(q)$,

(2) $\Phi_{*}(\{i\}) \cap \Phi_{*}(\{j\}) \subseteq A_{m(B)}$ for all distinct $i$ and $j$ contained in $\operatorname{dom}(q)$.

To see that we can ensure (1), note that since $\Phi_{h_{B}}$ is an almost lifting of $\Phi^{B}, B \cap \Phi_{h_{B}}(s)={ }_{\mathcal{I}} \Phi_{*}(s)$ for all finite $s$.

Since $\left(\left[\omega_{1}\right]^{\aleph_{0}}, \subseteq^{*}\right)$ is $\sigma$-directed, there is an $\bar{m} \geq \bar{n}$ such that $\mathcal{X}_{1}=\{B \in$ $\left.\mathcal{X}: \bar{m}=m_{B}\right\}$ is cofinal in $\left(\left[\omega_{1}\right]^{\aleph_{0}}, \subseteq^{*}\right)$ (see e.g. [Far00, Lemma 2.2.2]).

Claim 4. The set $\mathcal{X}_{1}$ is $K_{1}^{\bar{m}}$-homogeneous. 
Proof. We need to prove that the functions $h_{B} \uparrow\left(B \backslash A_{\bar{m}}\right)$ for $B \in \mathcal{X}_{1}$ are pairwise compatible. (We say that two functions are compatible if they agree on the intersection of their domains.) Assume not, and fix $A, B$ in $\mathcal{X}_{1}$ and $\xi \in(A \cap B) \backslash A_{\bar{m}}$ such that $i=h_{A}(\xi) \neq h_{B}(\xi)=j$.

Assume for a moment that $\{i, j\} \subseteq \operatorname{dom}(q)$. By (1), we have $\xi \in \Phi_{*}(\{i\})$ and $\xi \in \Phi_{*}(\{j\})$, so $\Phi_{*}(\{i\}) \cap \Phi_{*}(\{j\}) \nsubseteq A_{\bar{m}}$, contradicting (2).

Therefore we must have $i \notin \operatorname{dom}(q)$ or $j \notin \operatorname{dom}(q)$, and in either case we can find $r \leq q$ such that

$$
r \Vdash|\dot{C} \cap\{i, j\}|=1 .
$$

But then $r$ forces that $\chi_{\dot{C}} \circ h_{B}(\xi) \neq \chi_{\dot{C}} \circ h_{A}(\xi)$, and therefore that $\{A, B\} \in$ $L_{0}^{\bar{m}}(\dot{C})$, a contradiction.

By Claim 4 and Lemma 3.8, this concludes the proof modulo the assumption that some condition $p \in \mathcal{C}$ forces that there is $\bar{n}$ for which some $L_{1}^{\bar{n}}(\dot{C})$ homogeneous set $\dot{\mathcal{H}}$ is cofinal in $\left(\left(\left[\omega_{1}\right]^{\aleph_{0}}\right)^{V}, \subseteq^{*}\right)$. So assume that this fails, and $\mathcal{C}$ forces that a cofinal $L_{1}^{n}(\dot{C})$-homogeneous subset of $\left(\left(\left[\omega_{1}\right]^{\aleph_{0}}\right)^{V}, \subseteq^{*}\right)$ does not exist for any $n \in \omega$. The proof of Theorem 3.6 will be completed once we prove that this assumption leads to a contradiction.

Recall that for every $\delta<\omega_{1}$ there is a partial map

$$
h_{\delta}: \delta \rightarrow \omega
$$

such that $\Phi_{h_{\delta}}$ is a completely additive almost lifting of $\Phi^{\delta}$.

Let $C$ be a Cohen-generic subset of $\omega$ over $V$. In $V[C]$ we will define a ccc poset $\mathcal{P}$ that forces an uncountable $\mathcal{Z} \subseteq \omega_{1}$ and for each $\xi \in \mathcal{Z}$ a $B_{\xi} \subseteq \xi$ such that $B_{\xi} \cap A_{n}$ is finite for all $n$ and the functions in $\left\{h_{\xi}\left\lceil B_{\xi}: \xi \in \mathcal{Z}\right\}\right.$ are pairwise incompatible.

A typical condition $p$ of $\mathcal{P}$ is a triple $\left(F, n,\left\langle s_{\xi}: \xi \in F\right\rangle\right)$ such that

$(\mathcal{P} 0) F$ is a finite set of countable ordinals,

$(\mathcal{P} 1) n \in \omega$,

$(\mathcal{P} 2) s_{\xi}$ is a finite subset of $A_{n} \cap \xi$,

$(\mathcal{P} 3)$ the functions $\chi_{C} \circ h_{\xi}\left\lceil s_{\xi}(\xi \in F)\right.$ are pairwise incompatible.

The ordering on $\mathcal{P}$ is defined by letting $p \leq q$ if $F^{p} \supseteq F^{q}, n^{p} \geq n^{q}$, and

$(\mathcal{P} 4) s_{\xi}^{p} \cap A_{n^{q}}=s_{\xi}^{q}$ for all $\xi \in F^{q}$.

If $G$ is a sufficiently generic filter of $\mathcal{P}$, then for $\xi \in \mathcal{Z}=\bigcup_{q \in G} F^{q}$ the set $B_{\xi}=\bigcup_{p \in G} s_{\xi}^{p}$ is orthogonal to $\mathcal{I}$, and the family of $g_{\xi}=h_{\xi} \backslash B_{\xi}(\xi \in$ $\left.\bigcup_{p \in G} F^{p}\right)$ are such that $\chi_{C} \circ g_{\xi}$ are pairwise incompatible. Since $B_{\xi} \subseteq \xi, \Phi_{g_{\xi}}$ is an almost lifting of $\Phi^{B_{\xi}}$.

Claim 5. The poset $\mathcal{P}$ is ccc. 
Proof. Let $p_{\alpha}\left(\alpha<\omega_{1}\right)$ be an uncountable subset of $\mathcal{P}$. By going to an uncountable subset, we can assume that the sets $F^{\alpha}=F^{p_{\alpha}}$ form a $\Delta$-system with root $\bar{F}$, that all $n^{\alpha}=n^{p_{\alpha}}$ are equal to some fixed $\bar{n}$, and that for some $\bar{s}_{\xi}(\xi \in \bar{F})$ and all $\alpha$ we have $s_{\xi}^{\alpha}=\bar{s}_{\xi}$. By Lemma 3.7, for each $\alpha$ there is an $m^{\alpha} \geq \bar{n}$ large enough so that $\{\xi, \eta\} \in K_{1}^{m^{\alpha}}$ for all $\xi, \eta \in F^{\alpha}$. Find $\bar{m}$ such that $m^{\alpha}=\bar{m}$ for uncountably many $\alpha$. For each of these $\alpha$ let

$$
\xi(\alpha)=\min \left(F^{\alpha} \backslash \bar{F}\right) .
$$

The set of all $\xi(\alpha)$ is cofinal in $\left[\omega_{1}\right]^{\aleph_{0}}$, and it is therefore not $L_{1}^{\bar{m}}(C)$ homogeneous, by our assumption. Therefore there are $\alpha \neq \beta$ and $\gamma \in$ $(\xi(\alpha) \cap \xi(\beta)) \backslash A_{\bar{m}}$ such that $h_{\xi(\alpha)}(\gamma) \in C$ and $h_{\xi(\beta)}(\gamma) \notin C$. Let $i>\bar{m}$ be such that $\gamma \in A_{i}$. Define a condition $q$ as follows: Let $F^{q}=F^{\alpha} \cup F^{\beta}$, $n^{q}=i+1, s_{\xi}^{q}=\bar{s}_{\xi}$ for $\xi \in \bar{F}$, and $s_{\xi}^{q}=s_{\xi}^{\delta} \cup\{\gamma\}$ for $\xi \in F^{\delta} \backslash \bar{F}$ (if $\delta \in\{\alpha, \beta\}$ ).

For all $\eta \in F^{\alpha} \backslash \bar{F}$ we have

$$
h_{\eta}(\gamma)=h_{\xi(\alpha)}(\gamma) \in C
$$

and for all $\eta \in F^{\beta} \backslash \bar{F}$ we have

$$
h_{\eta}(\gamma)=h_{\xi(\beta)}(\gamma) \notin C,
$$

so the functions $\chi_{C} \circ h_{\eta}\left\lceil s_{\eta}^{q}\left(\eta \in F^{q}\right)\right.$ are pairwise incompatible.

Therefore $q$ extends both $p^{\alpha}$ and $p^{\beta}$. This proves that $\mathcal{P}$ is ccc.

Since $\mathcal{P}$ is ccc, some $p \in \mathcal{P}$ forces that $\mathcal{Z}=\bigcup_{q \in G} F^{q}$ is uncountable. By applying MA to $\mathcal{C} * \mathcal{P}$ and an appropriate family of dense sets, we get $C \subseteq \omega$, an uncountable $\mathcal{Z} \subseteq \omega_{1}$, and pairs $\left(B_{\xi}, g_{\xi}\right)(\xi \in \mathcal{Z})$ such that $B_{\xi} \in \mathcal{J}, g_{\xi}: B_{\xi} \rightarrow \omega$, and $\Phi_{g_{\xi}}$ is an almost lifting of $\Phi^{B_{\xi}}$ for all $\xi \in \mathcal{Z}$, and moreover the functions $\chi_{C} \circ g_{\xi}\left(\xi<\omega_{1}\right)$ are pairwise incompatible. If the partition $L_{0}^{0}(C)$ is re-evaluated using functions $g_{\xi}$ in place of $h_{B_{\xi}}$, the set $\left\{B_{\xi}: \xi \in \mathcal{Z}\right\}$ is $L_{0}^{0}(C)$-homogeneous. But this contradicts (c) of Lemma 3.9. This concludes the proof of Theorem 3.6.

The Cohen poset was used in a similar context of liftings by ChristensenKanovei-Reeken ([CKR01]), Kanovei-Reeken ([Far04, §8]), and Veličković ([Vel93, Theorem 4.1]). The first two references apply forcing to Borel liftings, while Veličković proved that under MA and OCA all automorphisms of $\mathcal{P}\left(\omega_{1}\right) /$ fin are trivial using, instead, the forcing for adding $\aleph_{1}$ side-by-side Cohen reals.

Corollary 3.10. Assume $O C A$ and $M A$. If $\mathcal{B}$ is a subalgebra of $\mathcal{P}\left(\omega_{1}\right)$ such that $\mathcal{P}(\omega)$ is a subalgebra of $\mathcal{B} / \mathcal{I}$ for some countably generated ideal $\mathcal{I}$, then $\mathcal{P}(\omega)$ is a subalgebra of $\mathcal{B}$.

Proof. If $\Psi: \mathcal{P}(\omega) \rightarrow \mathcal{B} / \mathcal{I}$ is a monomorphism, then it is also a lifting of a homomorphism $\Phi: \mathcal{P}(\omega) \rightarrow \mathcal{P}\left(\omega_{1}\right) / \mathcal{I}_{0}$, where $\mathcal{I}_{0}$ is an ideal on $\omega_{1}$ generated by $\mathcal{I}$. Let $\left\{I_{n}: n \in \omega\right\}$ be an increasing chain of members of $\mathcal{I}$ which 
generates $\mathcal{I}_{0}$. Let $h: \omega_{1} \rightarrow \omega$ be such that $\Phi_{h}$ is an $\aleph_{2}$-almost lifting of $\Phi$. Thus, if $A_{\xi}(\xi<\mathfrak{c})$ is any almost disjoint family of infinite subsets of $\omega$, then there is a member of the family, say $A_{0}$, such that $\Psi(B)={ }_{\mathcal{I}_{0}} \Phi_{h}(B)$ for all $B \subseteq A_{0}$. For each such $B$, we may let $n_{B} \in \omega$ be minimal such that $\Phi_{h}(B) \backslash I_{n_{B}} \in \mathcal{B}$. We will now prove there is an infinite subset $A$ of $A_{0}$ and an integer $m$ such that $n_{B} \leq m$ for all $B \subset A$. Fix any pairwise disjoint family of infinite subsets of $A_{0}$, say $\left\{C_{k}: k \in \omega\right\}$, and assume that for each $k$, there is a $B_{k} \subset C_{k}$ such that $n_{B_{k}}>n_{k}=\max \left\{k, n_{C_{k}}\right\}$. Fix any $B \subset A_{0}$ such that $B \cap C_{k}$ is almost equal to $B_{k}$ for each $k$ and note that $\Phi_{h}(B) \backslash I_{n_{k}} \cap \Phi_{h}\left(C_{k}\right) \backslash I_{n_{k}}$ is not in $\mathcal{B}$ for each $k$. For $k \geq n_{B}$, this contradicts the fact that $\Phi_{h}(B) \backslash I_{n_{B}} \in \mathcal{B}$.

The proof is completed then by observing that the following is an embedding of $\mathcal{P}(A)$ into $\mathcal{B}$. For each $B \subset A$, define $f(B)$ to be $\Phi_{h}(B) \backslash I_{m}$ if $\min A \notin B$ and to be $\Phi_{h}(B) \cup I_{m}$ if $\min A \in B$.

The following is a topological restatement of Corollary 3.10.

Corollary 3.11. Assume OCA and MA. If a compact zero-dimensional space $X$ has density at most $\omega_{1}$, and has a closed $G_{\delta}$ set which maps onto $\beta \mathbb{N}$, then $X$ itself maps onto $\beta \mathbb{N}$.

The Weak Extension Principle was studied in [Far00, Chapter 4]. The dual of Theorem 3.2 is its special case. The following corollary has a similar flavor.

Corollary 3.12. If $G$ is a closed $G_{\delta}$ subset of $\beta \omega_{1}$ and $f: G \rightarrow \beta \omega$ is continuous, then there is a clopen $U \subseteq G$ such that $f\lceil U$ continuously extends to $\beta \omega_{1}$ and $f^{\prime \prime}(G \backslash U)$ is nowhere dense.

Proof. Let $\mathcal{I}$ be the countably generated ideal on $\omega_{1}$ such that $G=\{p \in$ $\left.\beta \omega_{1}: p \cap \mathcal{I}=\emptyset\right\}$. Apply Theorem 3.6 to the homomorphism $\Phi: \mathcal{P}(\omega) \rightarrow$ $\mathcal{P}\left(\omega_{1}\right) / \mathcal{I}$ dual to $f$ to obtain $h$. Let $C=\operatorname{dom}(h)$ and $U=\beta C \cap G$. Then the continuous extension of $h$ : $C \rightarrow \omega$ to a map from $\beta C$ to $\beta \omega$ extends $f\lceil U$. The image of $G \backslash U$ is included in the set

$$
X=\left\{p \in \beta \omega: p \cap \operatorname{ker}\left(\Phi^{\omega_{1} \backslash C}\right)=\emptyset\right\} .
$$

This set has the property that every family of pairwise disjoint open subsets of $\omega^{*}$ each of which intersects $X$ has size at most $\aleph_{1}<\mathfrak{c}$, and it is therefore nowhere dense.

In [Far00] it was conjectured that the statement "every continuous $f: \omega^{*}$ $\rightarrow \beta \omega$ continuously extends to $\beta \omega$ " is consistent with ZFC.

QuESTION 3.13. Is the following consistent with $Z F C:$ If $G$ is a closed $G_{\delta}$ subset of some $\beta \kappa$ then every continuous $f: G \rightarrow \beta \omega$ continuously extends to $\beta \kappa$ ? 
It may be worth mentioning that Veličković has proved that under PFA every automorphism of any $\kappa^{*}$ continuously extends to a function from $\beta \kappa$ to $\beta \kappa$ ([Vel93]). A survey of some related extension principles and conjectures (or rather their algebraic duals) for quotients $\mathcal{P}(\omega) / \mathcal{I}$ can be found in $[\operatorname{Far} \infty]$.

4. Limitations. We shall now see that Theorem 3.6 cannot be further improved by replacing $\omega_{1}$ with $\mathfrak{c}$. Let us say that $\mathcal{A}$ is completely embeddable into $\mathcal{B}$ if there is a completely additive monomorphism $\Phi: \mathcal{A} \rightarrow \mathcal{B}$, that is, such that $\Phi(\bigvee \mathcal{X})=\bigvee_{a \in \mathcal{X}} \Phi(a)$ for every $\mathcal{X} \subseteq \mathcal{A}$. Let us state a slight strengthening of Corollary 3.10.

Corollary 4.1. Assume $O C A$ and $M A$. If $\mathcal{B}$ can be embedded into $\mathcal{P}\left(\omega_{1}\right)$, then the following are equivalent:

(1) $\mathcal{P}(\omega)$ embeds into $\mathcal{B}$.

(2) $\mathcal{P}(\omega)$ completely embeds into $\mathcal{B}$.

(3) $\mathcal{P}(\omega)$ embeds into $\mathcal{B} / \mathcal{I}$ for some countably generated ideal $\mathcal{I}$ on $\mathcal{B}$.

Proof. The only nontrivial implication is (3) implies (2), and it is an immediate consequence of Theorem 3.6 and the embedding as defined in Corollary 3.10.

Proposition 4.2. There is a subalgebra $\mathcal{B}$ of $\mathcal{P}(\mathfrak{c})$ such that $\mathcal{P}(\omega)$ is embeddable, but not completely embeddable, into $\mathcal{B}$.

Proof. Let $X$ be any countably compact dense subset of $\beta \omega$ such that $X$ has cardinality $\mathfrak{c}$ (see [Nov53]). It is easy to see that every infinite closed subset of $X$ has cardinality $\mathfrak{c}$.

We let $B$ denote the algebra generated by the clopen subsets of $X$ together with the singletons. Equivalently, $B$ is the algebra of all sets of the form ( $\bar{A}$ is the closure of $A$ in $\beta \omega$ intersected with $X$ )

$$
H_{0} \cup\left(\bar{A} \backslash H_{1}\right)
$$

for $A \subset \omega$ and finite subsets $H_{0}, H_{1}$ of $X$. To see this, note that all sets of the above form are in $B$ and that such sets form a Boolean algebra.

The mapping $\Phi(A)=\bar{A}$ is an embedding of $\mathcal{P}(\omega)$ into $B$. Assume there is a complete embedding $\Psi: \mathcal{P}(\omega) \rightarrow B$. Let $\Psi(\{n\})=b_{n}$. We can fix the sets $A_{n} \subset \omega$ and the finite sets $H_{0}^{n}, H_{1}^{n}$ such that $b_{n}=H_{0}^{n} \cup\left(\bar{A}_{n} \backslash H_{1}^{n}\right)$. We may of course assume that $H_{1}^{n}$ is disjoint from $\omega$. Note that the sets $A_{m}$ $(m \in \omega)$ are pairwise disjoint.

For now we can consider $c_{n}=H_{0}^{n} \cup \bar{A}_{n}$ and points $x \in X$ which are limits of the family $\left\{c_{n}: n \in \omega\right\}$ and which are not in $\bigcup_{n} b_{n}$ or in $\bigcup_{n} H_{0}^{n}$. Since there are $\mathfrak{c}$ such limits, such an $x$ exists.

Then $\Psi(\omega)=X=\bigvee_{n} b_{n}$. But $\{x\} \wedge \bigvee_{n} b_{n}=0_{B}$, a contradiction. 
Let us note that Theorem 3.6 cannot be improved by replacing $\aleph_{2}$-cc with ccc.

Proposition 4.3. There is a homomorphism $\Phi: \mathcal{P}(\omega) \rightarrow \mathcal{P}\left(\omega_{1}\right)$ with no completely additive almost lifting.

Proof. Let $A_{\xi}\left(\xi<\omega_{1}\right)$ be an almost disjoint family of infinite subsets of $\omega$ and let, for each $\xi \in \omega_{1}, \mathcal{U}_{\xi} \ni A_{\xi}$ be a nonprincipal ultrafilter. Define $\Phi: \mathcal{P}(\omega) \rightarrow \mathcal{P}\left(\omega_{1}\right)$ by

$$
\Phi(C)=\left\{\xi: C \in \mathcal{U}_{\xi}\right\} .
$$

Then $\operatorname{ker}(\Phi) \supseteq \mathcal{P}(\omega) \backslash \bigcup_{\xi} \mathcal{U}_{\xi}$. The mapping $F: \mathcal{P}(\omega) \rightarrow \mathcal{P}\left(\omega_{1}\right)$ defined by $F(C)=\emptyset$ is a lifting of $\Phi$ on the ideal $\operatorname{ker}(\Phi)$, but this ideal is not ccc over fin (as the sets $A_{\xi}$ witness).

However, if $h: \omega_{1} \rightarrow \omega$ is any partial function with non-empty domain, then it is easy to see that $\Phi_{h}$ is also not an almost lifting of $\Phi$. Indeed, fix any $\alpha \in \operatorname{dom}(h)$ and almost disjoint subsets of $\omega,\left\{B_{\xi}: \xi \in \omega_{1}\right\}$, such that $h(\alpha) \in B_{\xi}$ for each $\xi$ and $B_{\xi} \notin \mathcal{U}_{\eta}$ for all $\eta$. By construction $\Phi\left(B_{\xi}\right)$ is $\emptyset$ for all $\xi$, and yet $\alpha \in h^{-1}\left(B_{\xi}\right)=\Phi_{h}\left(B_{\xi}\right)$ for all $\xi$.

\section{References}

[BSS96] M. Bell, L. Shapiro and P. Simon, Products of $\omega^{*}$ images, Proc. Amer. Math. Soc. 124 (1996), 1593-1599.

[Bla73] A. Blass, The Rudin-Keisler ordering of P-points, Trans. Amer. Math. Soc. 179 (1973), 145-166.

[CKR01] J. P. R. Christensen, V. Kanovei and M. Reeken, On Borel orderable groups, Topology Appl. 109 (2001), 285-299.

[Dow97] A. Dow, Is $\mathcal{P}(\omega)$ a subalgebra?, preprint, 1997; available at http://www. math.uncc.edu/ adow/Bell_CH/Bell_CH.pdf.

[Far00] I. Farah, Analytic quotients: theory of liftings for quotients over analytic ideals on the integers, Mem. Amer. Math. Soc. 148 (2000), no. 702.

[Far04] —, Luzin gaps, Trans. Amer. Math. Soc. 356 (2004), 2197-2239.

[Far $\infty] \quad-$, Rigidity conjectures, in: Proc. Logic Colloq. 2000, to appear; available at http://www.math.yorku.ca/ ifarah.

[Jus89] W. Just, The space $\left(\omega^{*}\right)^{n+1}$ is not always a continuous image of $\left(\omega^{*}\right)^{n}$, Fund. Math. 132 (1989), 59-72.

[Kop89] S. Koppelberg, Handbook of Boolean Algebras, Vol. 1, J. D. Monk and R. Bonnet (eds.), North-Holland, Amsterdam, 1989,

[vM01] J. van Mill, On Dow's solution of Bell's problem, Topology Appl. 111 (2001), 191-193.

[Nov53] J. Novák, Über die bikompakte Hülle einer isolierten abzählbaren Menge, in: Bericht über die Mathematiker-Tagung in Berlin, Januar, 1953, Deutscher Verlag Wiss., Berlin, 1953, 280-283.

[Tod89] S. Todorčević, Partition Problems in Topology, Contemp. Math. 84, Amer. Math. Soc., Providence, RI, 1989. 
[Vel93] B. Veličković, OCA and automorphisms of $\mathcal{P}(\omega)$ /fin, Topology Appl. 49 (1993), $1-13$.

Department of Mathematics

UNC-Charlotte

9201 University City Blvd.

Charlotte, NC 28223-0001, U.S.A.

E-mail: adow@uncc.edu

http://www.math.uncc.edu/ adow
Department of Mathematics and Statistics York University 4700 Keele Street

North York, Ontario, Canada, M3J 1P3

E-mail: ifarah@mathstat.yorku.ca http://www.math.yorku.ca/ ifarah

and

Matematicki Institut

Kneza Mihaila 35

Beograd, Serbia and Montenegro

Received 6 September 2002;

in revised form 29 September 2004 